\title{
Dark energy from modified gravity with Lagrange multipliers
}

\author{
Salvatore Capozziello ${ }^{1,2}$, Jiro Matsumoto ${ }^{3}$, Shin'ichi Nojiri ${ }^{3,4}$ and Sergei D. Odintsov ${ }^{5 *}$ \\ 1 Dipartimento di Scienze Fisiche Universit'a "Federico II" di Napoli and ${ }^{2}$ INFN Sez. \\ di Napoli Compl. Univ. Monte S. Angelo Ed. N, via Cinthia I-80126 Napoli, Italy \\ 3 Department of Physics, Nagoya University, Nagoya 464-8602, Japan \\ ${ }^{4}$ Kobayashi-Maskawa Institute for the Origin of Particles and the Universe, Nagoya University, Nagoya 464-8602, Japan \\ ${ }^{5}$ Instituciò Catalana de Recerca i Estudis Avançats (ICREA) and Institut de Ciencies de l'Espai (IEEC-CSIC), \\ Campus UAB, Facultat de Ciencies, Torre C5-Par-2a pl, E-08193 Bellaterra (Barcelona), Spain
}

\begin{abstract}
We study scalar-tensor theory, k-essence and modified gravity with Lagrange multiplier constraint which role is to reduce the number of degrees of freedom. Dark Energy cosmology of different types $(\Lambda \mathrm{CDM}$, unified inflation with DE, smooth non-phantom/phantom transition epoch) is reconstructed in such models. It is demonstrated that presence of Lagrange multiplier simplifies the reconstruction scenario. It is shown that mathematical equivalence between scalar theory and $F(R)$ gravity is broken due to presence of constraint. The cosmological evolution is defined by the second $F_{2}(R)$ function dictated by the constraint. The convenient $F(R)$ gravity sector is relevant for local tests. This opens the possibility to make originally non-realistic theory to be viable by adding the corresponding constraint. A general discussion on the role of Lagrange multipliers to make higher-derivative gravity canonical is developed.
\end{abstract}

PACS numbers: 95.36. $+\mathrm{x}, 98.80 . \mathrm{Cq}$

\section{INTRODUCTION}

The understanding of late-time cosmic acceleration (the so-called Dark Energy (DE) epoch) is one of the main challenges of modern cosmology. There is no yet totally convincing theoretical DE model which is related also with the lack of precise observational data. This requests the introduction of new DEs in order to present fundamental and simple DE. It seems that standard strategy of combination of known models does not work and some new approach should be invented. Recently, a quite interesting new DE model [1, 2] was proposed which consists of two scalar fields where one of scalars represents the Lagrange multiplier. This multiplier puts the constraint of special form on the second scalar field. As a result, the whole system contains the single dynamical degree of freedom. It was shown that energy of such theory flows along time-like geodesics which is similar to the dust. Nevertheless, the theory contains non-zero pressure (dusty dark energy) [1, 2]. It may be very natural candidate for unification of Dark Energy and Dark Matter. It is interesting to study the role of such novel construction in the known DE models because it may completely change the structure of theory, its cosmological solutions and cosmological perturbations theory and may give better and/or qualitatively different fit towards to observational data. Moreover, the role of Lagrange multipliers may be twofold because they are often used for reducing of higher-derivative gravity systems in canonical formulation. Hence, the most natural area for the study of Lagrange multiplier constraint is modified gravity. In addition, adding such constraint in modified gravity may significally improve the ultraviolet properties of the covariant theory [3] leading to its renormalizability conjecture.

In the present letter we discuss the role of such Lagrange multiplier constraint in modified gravity. This is the first study of this sort, so the number of models are discussed in order to understand the general role of Lagrange multiplier construction and its impact to cosmology. We start from unified (phantom/canonical) scalar theory with Lagrange multiplier constraint. The reconstruction scenario for such theory as well as for k-essence theory is developed and shown to be significally simpler than in the case without constraint. Dark Energy cosmology of different types is reconstructed including standard $\Lambda$ CDM, unified inflation-Dark Energy era and non-phantom/phantom transition cosmology which turns out to be smooth. The comparison with standard theory is done, it is shown that different potentials describe such cosmological evolution. Moreover, convenient scalar does not propagate and does not generate the extra force. Despite the fact that theory is described by single degree of freedom, it may be represented in BD-form where Lagrange multiplier has ghost-like kinetic term.

The relation of such theory with modified $F(R)$-gravity is investigated. It is shown that mathematical equivalence between scalar theory and $F(R)$-gravity is broken because of the presence of Lagrange multiplier constraint. New constrained form of modified gravity is introduced. It consists of standard $F_{1}(R)$-term and Lagrange multiplier which constrains kinetic-like term for curvature by second function $F_{2}(R)$. The cosmological dynamics of such modified

\footnotetext{
* Also at Tomsk State Pedagogical University
} 
gravity depends only from the choice of $F_{2}(R)$ function. Dark energy cosmology may be easily reconstructed using its form.

Unlike to scalar theory, the Lagrange multiplier propagates. Hence, the role of $F_{1}(R)$ is in modification of Newton law which may cause the extra modification of constraint in order to reproduce the standard Newton law. This is also novel property, as in convenient modified gravity if theory does not pass newton law regime it is ruled out as realistic one.

Another application of Lagrange multiplier in canonical formulation is discussed. General method of reduction of higher derivatives is developed. It can be adopted for any higher-order theories. Specifically, gravity theories of order $(2 k+4)$, with $k$ an integer, need $(k+1)$ Lagrange multipliers to be made canonical, as we will discuss below.

The layout of this letter is the following. In Sec.II, we study scalar-tensor and k-essence theories with Lagrange multiplier constraint. DE cosmologies which are generated by the self-interaction potential and Lagrange multiplier constrained fluid are investigated. Sec.III is devoted to the same issue but $F(R)$ gravity is considered. In this case, cosmological dynamics is qualitatively changed due to lack of mathematical equivalence with scalar-tensor theory and presence of second $F_{2}(R)$ function caused by Lagrange multiplier constraint. In Sec.IV, general considerations on Lagrange multipliers are developed. We show that a given gravity theory of order $(2 k+4)$ can be made canonical by introducing $(k+1)$ suitable Lagrange multipliers. Conclusions are drawn in Sec.V.

\section{DARK ENERGY IN SCALAR THEORY WITH LAGRANGE MULTIPLIER}

In this section, we consider the accelerating FRW cosmology in the theory with two scalars where one of scalars is Lagrange multiplier which constrains the field equation of second scalar. The starting action has the following form:

$$
S=\int d^{4} x \sqrt{-g}\left\{\frac{R}{2 \kappa^{2}}-\frac{\omega(\phi)}{2} \partial_{\mu} \phi \partial^{\mu} \phi-V(\phi)-\lambda\left(\frac{1}{2} \partial_{\mu} \phi \partial^{\mu} \phi+U(\phi)\right)\right\} .
$$

Here $\lambda$ is the Lagrange multiplier field. Depending on the sign of the potential $\omega(\phi)$ (see ref.[4]) the first scalar could be the canonical scalar or the phantom. The Einstein equation has the following form:

$$
\frac{1}{2 \kappa^{2}}\left(R_{\mu \nu}-\frac{1}{2} g_{\mu \nu} R\right)=\frac{1}{2} g_{\mu \nu}\left\{-\frac{\omega(\phi)}{2} \partial_{\rho} \phi \partial^{\rho} \phi-V(\phi)-\lambda\left(\frac{1}{2} \partial_{\rho} \phi \partial^{\rho} \phi+U(\phi)\right)\right\}+\frac{\omega(\phi)+\lambda}{2} \partial_{\mu} \phi \partial_{\nu} \phi .
$$

We now work in the FRW metric with flat spatial part:

$$
d s^{2}=-d t^{2}+a(t)^{2} \sum_{i=1,2,3}\left(d x^{i}\right)^{2} .
$$

Then by the variation over $\lambda$, we obtain

$$
0=\frac{\dot{\phi}^{2}}{2}-U(\phi)
$$

The FRW equations are given by

$$
\begin{aligned}
\frac{3}{\kappa^{2}} H^{2} & =\frac{\omega(\phi)+\lambda}{2} \dot{\phi}^{2}+V(\phi)+\lambda U(\phi)=(\omega(\phi)+2 \lambda) U(\phi)+V(\phi), \\
-\frac{1}{\kappa^{2}}\left(2 \dot{H}+3 H^{2}\right) & =\frac{\omega(\phi)+\lambda}{2} \dot{\phi}^{2}-V(\phi)-\lambda U(\phi)=\omega(\phi) U(\phi)-V(\phi) .
\end{aligned}
$$

These equations show that the EoS-parameter $w_{\phi \lambda}$ has the following form:

$$
w_{\phi \lambda}=\frac{\omega(\phi) U(\phi)-V(\phi)}{(\omega(\phi)+2 \lambda) U(\phi)+V(\phi)} .
$$

Eq.(44) can be integrated as

$$
t= \pm \int^{\phi} \frac{d \phi}{\sqrt{2 U(\phi)}} .
$$

as long as $U(\phi)$ is positive. Then by solving Eq. (77) with respect to $\phi$, one can find the $t$-dependence of $\phi: \phi=\phi_{0}(t)$. Substituting the expression of $\phi_{0}(t)$ into (5), we obtain a differential equation for $H$, which gives the $t$-dependence of 
$H: H=H_{0}(t)$. Finally substituting $\phi_{0}(t)$ and $H_{0}(t)$ into (5), we can find the $t$-dependence of the Lagrange multiplier field $\lambda$ :

$$
\lambda=-\frac{\omega\left(\phi_{0}(t)\right)}{2}+\frac{1}{2 U\left(\phi_{0}(t)\right)}\left\{\frac{3}{\kappa^{2}} H_{0}(t)^{2}-V\left(\phi_{0}(t)\right)\right\} .
$$

Note that due to the constraint (4), the dynamics is completely changed. Usually if the scalar field exists, the propagation of the field generates an extra force and often violates the observational constraints on the Newton law. Due to the constraint (4), however, there does not appear the propagating mode of $\phi$. To see this, we consider the perturbation from the solution $\phi_{0}(t)$ given by (7),

$$
\phi=\phi_{0}(t)+\delta \phi
$$

Here we assume that $\delta \phi$ can depend on both of the time coordinate $t$ and the spatial coordinates. Then the constraint equation (4) gives,

$$
0=\frac{d \delta \phi}{d t}-\frac{U^{\prime}(\phi)}{\frac{d \phi_{0}}{d t}} \delta \phi
$$

When $U^{\prime}(\phi) / \frac{d \phi_{0}}{d t}>0$, the perturbation $\delta \phi$ grows with time and therefore the solution $\phi_{0}$ becomes unstable. On the other hand, when $U^{\prime}(\phi) / \frac{d \phi_{0}}{d t}<0$, the solution $\phi_{0}$ becomes stable. Anyway there does not appear the oscillating mode in $\delta \phi$ and therefore $\delta \phi$ does not propagate nor does not generate the extra force. We should also note that in the equations (4) and (5), there does not appear the term containing the derivatives of $\lambda$, which shows that the multiplier field does not propagate. We now consider the reconstruction, that is, when the behavior of the Hubble rate $H$ : $H=H_{0}(t)$ is known, how can we construct the action in the form of (1) to reproduce $H_{0}(t)$. First we choose $U(\phi)$ appropriately so that we can easily integrate Eq. (7) to find explicit form of $\phi_{0}(t)$ and $t=t(\phi)$. Then Eq. (5) gives

$$
V(\phi)=\frac{1}{\kappa^{2}}\left(2 \dot{H}_{0}(t(\phi))+3 H_{0}(t(\phi))^{2}\right)+\omega(\phi) U(\phi) .
$$

Here $\omega(\phi)$ can be arbitrary. Hence, one finds that choosing $V(\phi)$ as (11), the arbitrary Hubble rate $H=H_{0}(t)$ can be reproduced.

Some examples may illustrate the reconstruction scheme. Just for simplicity, we assume

$$
\omega(\phi)=1, \quad U(\phi)=\frac{m^{4}}{2} .
$$

Here $m$ is a constant with the dimension of mass and canonical scalar is considered. Then Eq. (7) tells

$$
\phi=m^{2} t
$$

Here we have chosen the $+\operatorname{sign}$ in \pm in (7). Then Eq. (11) has the following form:

$$
V(\phi)=\frac{1}{\kappa^{2}}\left\{2 \dot{H}_{0}\left(\frac{\phi}{m^{2}}\right)+3 H_{0}\left(\frac{\phi}{m^{2}}\right)^{2}\right\}+\frac{m^{4}}{2} .
$$

Eq. (8) indicates that

$$
\lambda=-1-\frac{2}{\kappa^{2}} \dot{H}_{0}\left(\frac{\phi}{m^{2}}\right) .
$$

Let us consider $H_{0}(t)$ corresponding to the $\Lambda$ CDM model:

$$
H_{0}(t)=\frac{2}{3 l} \operatorname{coth}\left(\frac{t}{l}\right) .
$$

Here $l$ is the length parameter related with the cosmological constant. The potential $V(\phi)$ becomes a constant

$$
V(\phi)=\frac{4}{3 l^{2} \kappa^{2}}+\frac{m^{4}}{2},
$$


which may be regarded as a cosmological constant. The scalar field plays the role of dust [1, 2]. Thus, one obtains dark energy produced by dusty Lagrange multiplier field. Following the proposal of ref.[4], one can reconstruct the model unifying the inflation and the late-time acceleration:

$$
H_{0}(t)=\frac{H_{I}+H_{L} \frac{t^{2}}{t_{0}^{2}}}{1+\frac{t^{2}}{t_{0}^{2}}}
$$

When $t \rightarrow 0, H$ behaves as $H=H_{I}+\mathcal{O}\left(t^{2}\right)$. Then the universe is almost de Sitter space corresponding to the inflation. On the other hand, when $t \rightarrow \infty, H$ behaves as $H=H_{L}+\mathcal{O}\left(t^{-2}\right)$ and the universe becomes asymptotically de Sitter space describing dark energy era. Applying the above procedure one gets:

$$
V(\phi)=\frac{1}{\kappa^{2}} \frac{-4\left(H_{I}-H_{L}\right) \frac{\phi}{m^{2} t_{0}^{2}}+3\left(H_{I}+H_{L} \frac{\phi^{2}}{m^{4} t_{0}^{2}}\right)^{2}}{\left(1+\frac{\phi^{2}}{m^{4} t_{0}^{2}}\right)}+\frac{m^{4}}{2} .
$$

As a final example, we consider the dark energy model which admits the transition [5] from non-phantom phase to phantom phase:

$$
H_{0}(t)=h_{0}\left(\frac{1}{t}+\frac{1}{t_{s}-t}\right)
$$

Then we find

$$
V(\phi)=\frac{1}{\kappa^{2}}\left(\frac{m^{4}\left(3 h_{0}^{2}-2 h_{0}\right)}{\phi^{2}}+\frac{m^{4}\left(3 h_{0}^{2}-2 h_{0}\right)}{\left(m^{2} t_{s}-\phi\right)^{2}}+\frac{6 h_{0}^{2} m^{4}}{\phi\left(m^{2} t_{s}-\phi\right)^{2}}\right)+\frac{m^{4}}{2} .
$$

The theory with above scalar potential describes the transition from non-phantom phase to phantom era. The EoS parameter $w_{\phi \lambda}$ (6):

$$
w_{\phi \lambda}=-1-\frac{2 t-t_{s}}{3 h_{0} t_{s}}
$$

which shows the phantom crossing ( $w=-1$ crossing) when $t=t_{s} / 2$, that is, $w>-1$ when $t<t_{s} / 2$ and $w<-1$ when $t>t_{s} / 2$. Eq. (20) demonstrates that there is a Big Rip singularity at $t=t_{s}$ (for first works on Big Rip singularity, see [6]). To understand better the role of Lagrange multiplier constraint we consider single scalar-tensor theory [4, 7],

$$
S=\int d^{4} x \sqrt{-g}\left\{\frac{R}{2 \kappa^{2}}-\frac{\omega(\phi)}{2} \partial_{\mu} \phi \partial^{\mu} \phi-V(\phi)\right\} .
$$

The reconstruction can be performed by choosing

$$
\omega(\phi)=-\frac{2}{\kappa^{2}} H_{0}^{\prime}(\phi), \quad V(\phi)=\frac{1}{\kappa^{2}}\left(3 H_{0}(\phi)^{2}+H_{0}^{\prime}(\phi)\right) .
$$

Then it follows

$$
\phi=t, \quad H=f(t) .
$$

Especially in case of non-phantom/phantom transition (20), one gets[4]

$$
\omega(\phi)=h_{0}\left\{-\frac{1}{\phi^{2}}+\frac{1}{\left(t_{s}-\phi\right)^{2}}\right\}, \quad V(\phi)=\frac{1}{\kappa^{2}}\left(\frac{\left(3 h_{0}^{2}-h_{0}\right)}{\phi^{2}}+\frac{3 h_{0}^{2}-h_{0}}{\left(t_{s}-\phi\right)^{2}}+\frac{6 h_{0}^{2}}{\phi\left(t_{s}-\phi\right)^{2}}\right) .
$$

The form of the potential is similar to that in (21) but the coefficients are different from each other. We should also note that in (21), the mass dimension of the scalar field is unity as standard but that of the scalar field in (26) is minus unity by following [4, 7]. The resemblance of two potentials (21) and (26) comes from the expressions (11) or more explicitly (14) and (24). Note that in (14), the scalar field is chosen, essentially to be time coordinate as in (13) by the choice of $U(\phi)$ in (12). Expression (11) comes from the effective pressure but (24) comes from the difference between the effective pressure and the effective energy density. Then the coefficients in two potentials are different 
from each other. In spite of the similarity in the two models (23) and (1), there is a big difference in them, that is, the scalar in (23) propagates and often gives a correction to the Newton law but the scalars in (1) do not propagate and do not give any correction to the Newton law. Now both of the scalar-tensor theories with a constraint (21) and without a constraint (26) give the identical EoS parameter $w: w=w_{\phi \lambda}$ in (22). Then in both of the models, the phantom crossing occurs when $t=t_{s} / 2$ and there is a Big Rip singularity at $t=t_{s}$. We should note that $\omega(\phi)$ vanishes when the phantom crossing occurs at $t=t_{s} / 2$, which shows that if we redefine the scalar field as

$$
\varphi=\int d \phi \sqrt{|\omega(\phi)|}
$$

the action (23) can be rewritten as

$$
S=\int d^{4} x \sqrt{-g}\left\{\frac{1}{2 \kappa^{2}} R \mp \frac{1}{2} \partial_{\mu} \varphi \partial^{\mu} \varphi-\tilde{V}(\varphi)\right\}
$$

The sign in front of the kinetic term depends on the sign of $\omega(\phi)$. Therefore at the point of the phantom transition, the sign changes. In this sense, in the model (23), the phantom transition is not smooth and it has been shown that the transition is very unstable [4, 7]. On the other hand, for the model with a constraint (1), the transition seems to be smooth. Finally in this section, we show that the action (11) can be rewritten as the Brans-Dicke theory coupled with a (phantom) scalar field. Let $\omega(\phi=1$ and consider the conformal transformation

$$
g_{\mu \nu}=\mathrm{e}^{\sigma}, \quad \mathrm{e}^{\sigma / \sqrt{3}} \equiv(1+\lambda)^{-1}
$$

Then the action (11) has the following form:

$$
S=\int d^{4} x \sqrt{-g}\left\{\frac{\mathrm{e}^{\sigma / \sqrt{3}}}{2 \kappa^{2}}\left(R+\frac{1}{2} \partial^{\mu} \sigma \partial_{\mu} \sigma\right)-\frac{1}{2} \partial_{\mu} \phi \partial^{\mu} \phi-\mathrm{e}^{2 \sigma / \sqrt{3}} V(\phi)-\left(\mathrm{e}^{2 \sigma / \sqrt{3}}-\mathrm{e}^{\sigma / \sqrt{3}}\right) U(\phi)\right\} .
$$

Now the constraint disappears and the scalar field $\phi$ and the ex-Lagrange multiplier field $\lambda$ or $\sigma$ seems to be propagating. However, the kinetic term of $\sigma$ is not canonical (ghost-like). Then effectively the amplitude coming from the propagation of $\phi$ might be canceled by the propagation of $\sigma$. One may choose $V(\phi)$ so that the smooth phantom crossing occurs in our model. Thus, we demonstrated that the theory with two scalars where one of them is Lagrange multiplier may lead to variety of dark energy cosmologies. Despite the fact that the theory possesses single dynamical degree of freedom, its cosmology seems to be qualitatively different from the one of the theory with single scalar.

It is well-known that scalar-tensor theory is mathematically equivalent to $F(R)$ gravity which does not lead to physical equivalence of two theories[8]. Now we may try to transform the action (1) into the $F(R)$-gravity form. Making the conformal transformation

$$
g_{\mu \nu} \rightarrow \mathrm{e}^{\kappa \phi \sqrt{\frac{2}{3}}} g_{\mu \nu}
$$

the kinetic term of $\phi$ is canceled and one obtains

$$
S=\int d^{4} x \sqrt{-g}\left\{\frac{\mathrm{e}^{\kappa \phi \sqrt{\frac{2}{3}}} R}{2 \kappa^{2}}-\mathrm{e}^{2 \kappa \phi \sqrt{\frac{2}{3}}} V(\phi)-\lambda\left(\frac{1}{2} \mathrm{e}^{\kappa \phi \sqrt{\frac{2}{3}}} \partial_{\mu} \phi \partial^{\mu} \phi-\mathrm{e}^{2 \kappa \phi \sqrt{\frac{2}{3}}} U(\phi)\right)\right\} .
$$

In the standard case, there is no last term in the above action and scalar becomes the auxiliary one (no the kinetic term). Since the term containing $\lambda$ includes the derivative of $\phi$, we cannot integrate and/or delete the scalar field $\phi$ and therefore it is difficult to rewrite the action (1) in the $F(R)$-gravity form. Hence, the mathematical equivalence between two theories seems to be broken due to presence of the Lagrange multiplier term.

As an extension of the scalar field with constraint, we may consider k-essence model[9] with a constraint:

$$
S=\int d^{4} x \sqrt{-g}\left\{\frac{R}{2 \kappa^{2}}+K(\phi, X)+\lambda(X-U(\phi))+L_{\text {matter }}\right\}, \quad X \equiv-\frac{1}{2} \partial^{\mu} \phi \partial_{\mu} \phi,
$$

where $K$ is an appropriate function of $\phi$. Let us check if the introduction of Lagrange multiplier in such theory does not destroy its consistency and the reconstruction discussed in [10]. The FRW equations are given by

$$
\frac{3}{\kappa^{2}} H^{2}=2 X \frac{\partial K(\phi, X)}{\partial X}-K(\phi, X)+2 \lambda X+\rho_{\text {matter }}(t), \quad-\frac{1}{\kappa^{2}}\left(2 \dot{H}+3 H^{2}\right)=K(\phi, X)+p_{\text {matter }}(t) .
$$


Here we include the contribution of matters with a constant EoS parameters $w_{i}$. Then the energy density $\rho_{\text {matter }}$ and the pressure $p_{\text {matter }}$ of matters are given by

$$
\rho_{\text {matter }}=\sum_{i} \rho_{0 i} a^{-3\left(1+w_{i}\right)}, \quad p_{\text {matter }}=\sum_{i} w_{i} \rho_{0 i} a^{-3\left(1+w_{i}\right)} .
$$

Then the variation of (33) with respect to $\lambda$ gives,

$$
X-U(\phi)=0 .
$$

We choose $U(\phi)=m^{4} / 2$. So (36) gives $\phi=m^{2} t$, then one can rewrite the equations in (34) in the following form

$$
\begin{aligned}
& K\left(m^{2} t, m^{4} / 2\right)=-\frac{1}{\kappa^{2}}\left(2 \dot{H}+3 H^{2}\right)-\sum_{i} w_{i} \rho_{0 i} a^{-3\left(1+w_{i}\right)}, \\
& \left.\frac{\partial K\left(m^{2} t, X\right)}{\partial X}\right|_{X=m^{4} / 2}=-\frac{2}{\kappa^{2}} \dot{H}-\lambda-\sum_{i}\left(1+w_{i}\right) \rho_{0 i} a^{-3\left(1+w_{i}\right)} .
\end{aligned}
$$

Then by using an appropriate function $g\left(\phi / m^{2}\right)$, if we choose

$$
K\left(m^{2} t, m^{4} / 2\right)=-\frac{1}{\kappa^{2}}\left(2 g^{\prime \prime}\left(\phi / m^{2}\right)+3 g^{\prime}\left(\phi / m^{2}\right)^{2}\right)-\sum_{i} w_{i} \rho_{0 i} a_{0}^{-3\left(1+w_{i}\right)} \mathrm{e}^{-3\left(1+w_{i}\right) g\left(\phi / m^{2}\right)},
$$

one gets the following solution of (37):

$$
H=g^{\prime}(t) \quad\left(a=a_{0} \mathrm{e}^{g(t)}\right) .
$$

Note that $X$-dependence of $K(\phi, X)$ can be arbitrary as long as $K(\phi, X)$ satisfies (39). The $t$-dependence of $\lambda(t)$ can be determined by using (38) as

$$
\lambda=-\left.\frac{\partial K\left(m^{2} t, X\right)}{\partial X}\right|_{X=m^{4} / 2}-\frac{2}{\kappa^{2}} \dot{H}-\sum_{i}\left(1+w_{i}\right) \rho_{0 i} a^{-3\left(1+w_{i}\right)} .
$$

In case of $K(\phi, X)=K(X)$ action (33) has the following form:

$$
S=\int d^{4} x \sqrt{-g}\left\{\frac{R}{2 \kappa^{2}}+K(X)+\lambda(X-U(\phi))+L_{\text {matter }}\right\}, \quad X \equiv-\frac{1}{2} \partial^{\mu} \phi \partial_{\mu} \phi .
$$

If we set $U(\phi)=\frac{m^{3} \phi}{2}$, we find $\phi=m^{3} t^{2} / 2$ and therefore $U=X=m^{6} t^{2} / 2$. Then the second equation of (34) determines the form of $K(X)$ as follows,

$$
K(X)=-\frac{1}{\kappa^{2}}\left(2 \dot{H}\left(\sqrt{2 X / m^{6}}\right)+3 H^{2}\left(\sqrt{2 X / m^{6}}\right)\right)-\sum_{i} w_{i} \rho_{0 i} a\left(\sqrt{2 X / m^{6}}\right)^{-3\left(1+w_{i}\right)} .
$$

By differentiating the second equation of (34), we find

$$
m^{6} t K^{\prime}\left(m^{3} t^{2} / 2\right)=-\frac{1}{\kappa^{2}}(2 \ddot{H}+6 H \dot{H})+3 H \sum_{i} w_{i}\left(1+w_{i}\right) \rho_{0 i} a^{-3\left(1+w_{i}\right)} .
$$

Then combining (44) with the first equation of (34), the time dependence of $\lambda$ follows:

$$
\lambda=-\left(\frac{2 \dot{H}}{\kappa^{2}}+\sum_{i}\left(1+w_{i}\right) \rho_{0 i} a^{-3\left(1+w_{i}\right)}\right) \frac{1}{m^{6} t^{2}}+\left(\frac{2}{\kappa^{2}}(\ddot{H}+3 H \dot{H})-3 H \sum_{i} w_{i}\left(1+w_{i}\right) \rho_{0 i} a^{-3\left(1+w_{i}\right)}\right) \frac{1}{m^{6} t} .
$$

Using above technique the specific examples of DE cosmology may be reconstructed. Theory remains to be consistent but the reconstruction examples qualitatively change. This means that the same potential which was used to produce given cosmology leads to different cosmology in the presence of Lagrange multiplier. Moreover, explicit realization of reconstruction scenario turns out to be significally simpler than without constraint. 


\section{FRW COSMOLOGY IN $F(R)$-GRAVITY WITH LAGRANGE MULTIPLIER}

In this section we consider $F(R)$-gravity where Lagrange multiplier is introduced in the same way as in scalar theory of previous section. In the usual $F(R)$-gravity, there appears the scalar mode called scalaron, which often affects the Newton law. In this section, we try to suppress the propagation of the scalaron by imposing the constraint under the Lagrange multiplier field. As a result, however, there seems to appear the propagating mode in the Lagrange multiplier field, which may break the Newton law but in somehow easier way. The solution of this question may request the additional modification of constraint. Another purpose of this section is the reconstruction. In usual $F(R)$-gravity, we need to solve the complicated differential equation [8] to realize the reconstruction program. In this section, we show that the reconstruction can be done more easily in the model with the Lagrange multiplier field. The starting action is given by

$$
S=\int d^{4} x \sqrt{-g}\left\{F_{1}(R)-\lambda\left(\frac{1}{2} \partial_{\mu} R \partial^{\mu} R+F_{2}(R)\right)\right\} .
$$

Here $\lambda$ is the Lagrange multiplier field, again, which gives a constraint

$$
\frac{1}{2} \partial_{\mu} R \partial^{\mu} R+F_{2}(R)=0
$$

On the other hand, by the variation of the metric $g_{\mu \nu}$, we obtain an equation corresponding to the Einstein equation:

$$
0=\frac{1}{2} g_{\mu \nu} F_{1}(R)+\frac{\lambda}{2} \partial_{\mu} R \partial_{\nu} R+\left(-R_{\mu \nu}+\nabla_{\mu} \nabla_{\nu}-g_{\mu \nu} \nabla^{2}\right)\left(F_{1}^{\prime}(R)-\lambda F_{2}^{\prime}(R)-\nabla^{\mu}\left(\lambda \nabla_{\mu} R\right)\right) .
$$

If the Ricci curvature is covariantly constant and the scalar curvature is a constant:

$$
R_{\mu \nu}=\frac{R_{0}}{4} g_{\mu \nu}, \quad R=R_{0}
$$

Eqs. (47) and (48) reduce to

$$
\begin{aligned}
& 0=F_{2}\left(R_{0}\right) \\
& 0=F_{1}\left(R_{0}\right)-\frac{1}{2} R_{0}\left(F_{1}^{\prime}\left(R_{0}\right)-\lambda F_{2}^{\prime}\left(R_{0}\right)\right) .
\end{aligned}
$$

If Eq.(50) has a solution, Eq. (51) can be solved with respect to the Lagrange multiplier field:

$$
\lambda=\frac{-F_{1}\left(R_{0}\right)+R_{0} F_{1}^{\prime}\left(R_{0}\right)}{F_{2}^{\prime}\left(R_{0}\right)} .
$$

Then if $R_{0}$ is positive the above solution describes de Sitter space-time which may correspond to dark energy or inflationary epoch (for the proposal of gravitational unification of inflation with dark energy in modified gravity, see [11]). For spatially-flat FRW metric Eqs. (47) and $(\mu, \nu)=(0,0)$-component of (48) have the following form:

$$
\begin{aligned}
& 0=-\frac{1}{2} \dot{R}^{2}+F_{2}(R), \\
& 0=-\frac{1}{2} F_{1}(R)+18 \lambda(\ddot{H}+4 H \dot{H})^{2}+\left\{3\left(\dot{H}+H^{2}\right)-3 H \frac{d}{d t}\right\}\left\{F_{1}^{\prime}(R)-\lambda F_{2}^{\prime}(R)+\left(\frac{d}{d t}+3 H\right)\left(\lambda \frac{d R}{d t}\right)\right\} .
\end{aligned}
$$

When $F_{2}(R)>0$, Eq. (53) may be solved as

$$
t=\int^{R} \frac{d R}{\sqrt{2 F_{2}(R)}},
$$

which can be solved with respect to $R$ as a function of $t R=F_{R}(t)$. Since

$$
R=6 \frac{d H}{d t}+12 H^{2}
$$

one can find the behavior of $H=\frac{\dot{a}}{a}$ by solving the differential equation

$$
6 \frac{d H}{d t}+12 H^{2}=F_{R}(t),
$$


By using the obtained solution for $H=H(t)$ (and $R=F_{R}(t)$ ), Eq. (54) becomes a differential equation for the multiplier field $\lambda$ and we can find the behavior of $\lambda, \lambda=\lambda(t)$.

Conversely when the behavior of $H(t)$ is known from the observational data, one may reconstruct $F_{2}(R)$ to reproduce the behavior of $H(t)$ by using (53). $H(t)$ gives the behavior of $R$ as $R=R(t)$, which can be solved with respect to $t$ as $t=t(R)$. Using (53), the explicit form of $F_{2}(R)$ is found to be

$$
F_{2}(R)=\left.\frac{1}{2}\left(\frac{d R}{d t}\right)^{2}\right|_{t=t(R)}
$$

Note that $F_{1}(R)$ can be arbitrary function. Then the reconstruction of model can be more easily performed than that in the usual $F(R)$-gravity. As an explicit example, we may consider

$$
H(t)=\frac{h_{0}}{t}
$$

where $h_{0}$ is a constant. Then

$$
R=\frac{-6 h_{0}+12 h_{0}^{2}}{t^{2}} \quad \text { or } \quad t=\sqrt{\frac{-6 h_{0}+12 h_{0}^{2}}{R}} .
$$

And therefore, we find

$$
\frac{d R}{d t}=-\frac{12\left(-h_{0}+2 h_{0}^{2}\right)}{t^{3}}=-\frac{2 R^{\frac{3}{2}}}{\sqrt{6\left(-h_{0}+2 h_{0}^{2}\right)}}
$$

which gives

$$
F_{2}(R)=\frac{R^{3}}{12\left(-h_{0}+2 h_{0}^{2}\right)}
$$

Another example is given by

$$
R=\frac{R_{-}}{2}(1-\tanh \omega t)+\frac{R_{+}}{2}(1+\tanh \omega t) .
$$

Here $R_{ \pm}$and $\omega$ are constants. Then $t \rightarrow \pm \infty, R \rightarrow \pm R_{ \pm}$and therefore the space-time becomes asymptotically de Sitter. One may identify the epoch of $t \rightarrow-\infty$ as inflation and $t \rightarrow+\infty$ as late acceleration. Since

$$
\dot{R}=\frac{\left(R_{-}-R_{+}\right) \omega}{2 \cosh ^{2} \omega t}=\frac{\left(R_{-}-R_{+}\right) \omega}{2}\left(1-\frac{\left(R_{-}+R_{+}-2 R\right)^{2}}{\left(R_{-}-R_{+}\right)^{2}}\right)
$$

from Eq.(58), one gets

$$
F_{2}(R)=\frac{\left(R_{-}-R_{+}\right)^{2} \omega^{2}}{8}\left(1-\frac{\left(R_{-}+R_{+}-2 R\right)^{2}}{\left(R_{-}-R_{+}\right)^{2}}\right)^{2},
$$

Thus, the unification of early-time inflation with dark energy epoch is possible also in constraint modified gravity. Hence, the universe evolution only depends on the constraint equation (53) but does not depend on $F_{1}(R)$. $F_{1}(R)$ can only affect the correction to the Newton law. In convenient $F_{1}(R)$ cosmology the whole dynamics is defined by the form of this function. With the constraint (53),$F_{1}(R)$ becomes irrelevant. The cosmological dynamics is defined by the form of $F_{2}(R)$. In $F(R)$-gravity, there appears the propagating mode, which is often called scalaron and which often violates the Newton law. In the same way as in the scalar-tensor theory around (9), we may show that the scalaron does not propagate. In case of $F(R)$-gravity, however, Eq. (48) contains the second derivative of the multiplier field $\lambda$ although the Einstein equation (2) of the scalar theory with a constraint (1) does not contain the derivative of the multiplier field $\lambda$ and therefore $\lambda$ can be solved algebraically as in (8). In case of $F(R)$-gravity, we need to solve the second order differential equation to find $\lambda$, which might indicate that $\lambda$ could propagate and there might appear the correction to the Newton law. The magnitude of the correction could depend on the choice of $F_{1}(R)$ and/or $F_{2}(R)$. 
In order to investigate the Newton law, we choose $F_{1}(R)$ as the Einstein one,

$$
F_{1}(R)=\frac{R}{2 \kappa^{2}},
$$

and introduce the matter. Then Eq. (48) has the following form:

$$
0=\frac{1}{2 \kappa^{2}}\left(\frac{1}{2} g_{\mu \nu} R-R_{\mu \nu}\right)+\frac{1}{2} T_{\mu \nu}-\left(-R_{\mu \nu}+\nabla_{\mu} \nabla_{\nu}-g_{\mu \nu} \nabla^{2}\right)\left(\lambda F_{2}^{\prime}(R)-\nabla^{\mu}\left(\lambda \nabla_{\mu} R\right)\right) .
$$

For the solution where $\lambda=0$, Eq.(48) reduces to the Einstein equation,

$$
0=\frac{1}{\kappa^{2}}\left(\frac{1}{2} g_{\mu \nu} R-R_{\mu \nu}\right)+T_{\mu \nu} .
$$

Here $T_{\mu \nu}$ is the matter energy-momentum tensor. In the case without matter $T_{\mu \nu}=0$, the Schwarzschild space-time, where $R=R_{\mu \nu}=0$ is a solution, which also satisfies the constraint equation (47) if $F_{2}(0)=0$. In case with matter $T_{\mu \nu} \neq 0$, however, the Einstein equation (68) gives

$$
R=-\kappa^{2} T \text {. }
$$

Here $T$ is the trace of the energy-momentum tensor. The constraint equation (47) is rewritten to

$$
0=\frac{\kappa^{4}}{2} \partial_{\mu} T \partial^{\mu} T+F_{2}\left(-\kappa^{2} T\right)
$$

which is not always satisfied. Hence, in the presence of the matter, the constraint equation (47) should be modified to be

$$
0=\frac{1}{2} \partial_{\mu} R \partial^{\mu} R+F_{2}(R)-\frac{\kappa^{4}}{2} \partial_{\mu} T \partial^{\mu} T-F_{2}\left(-\kappa^{2} T\right) .
$$

This indicates that the total constrained action with matter should be, instead of 44,

$$
S=\int d^{4} x \sqrt{-g}\left[\frac{R}{2 \kappa^{2}}-\lambda\left\{\frac{1}{2} \partial_{\mu} R \partial^{\mu} R+F_{2}(R)-\frac{\kappa^{4}}{2} \partial_{\mu} T \partial^{\mu} T-F_{2}\left(-\kappa^{2} T\right)\right\}+\mathcal{L}_{\text {matter }}\right],
$$

In this case, the Newton law could be easily reproduced. Here $\mathcal{L}_{\text {matter }}$ is the Lagrangian of the matter. Of course, qualitatively other form of constraint may also solve this problem. We also note that the form of the constraint could be correct when $F_{1}(R)$ is given by (66). For general $F_{1}(R)$, the constraint could be changed.

Since $T$ vanishes in the vacuum as in the bulk of the universe, the constraint (71) reduces to

$$
0=\frac{1}{2} \partial_{\mu} R \partial^{\mu} R+F_{2}(R)-F_{2}(0) .
$$

If $F_{2}(0)=0$, the constraint (71) gives (47) and (53) and the cosmological evolution could be generated. Note that $F_{2}(R)$ in (62) satisfies the condition $F_{2}(0)=0$ but $F_{2}(R)$ in (65) does not. In case the condition $F_{2}(0)=0$ is satisfied, there are two classes of solution in the constraint (53). One is a trivial solution $R=0$ and another corresponds to the non-trivial cosmological evolution given by (55). Near the solar systems and the galaxies, the solution could correspond to the trivial one $R=0$ in order to reproduce the Newton law but in the bulk of the universe, the solution should correspond to (55) so that the evolution of the universe could be generated. It is not so trivial to show or to deny that the two solutions could be connected in the intermediate region between the region near the solar systems or galaxies and the region of the bulk universe. Maybe we need more careful (possibly numerical) analysis, which requests a future investigation in this direction.

Thus, we demonstrated the possibility to describe the cosmological dynamics, including dark energy era, in modified $F(R)$-gravity with Lagrange constraint. It turns out that reconstruction which produces the viable cosmology in this case is realized qualitatively easier than in the convenient modified gravity. Moreover, to pass the local tests may request the additional modifications of constraint equations as is seen for emergence of Newton law regime. This may be caused by the fact that Lagrange multiplier propagates in such a theory. It is quite interesting observation because modifying the form of constraint one may arrive to qualitatively different predictions about local tests, which can make the same theory with different constraint to be viable! 


\section{LAGRANGE MULTIPLIERS IN GENERIC HIGHER-ORDER THEORIES}

Let us discuss other application of Lagrange multiplies. In the optimization problems, such a method allows to find out extremal points (maxima and minima) of a function where one or more than one constraints are present. Specifically, Lagrange multipliers allow to calculate stationary points of the constrained function. In other words, the method reduces the search for stationary points of a $n$-variable function with $k$-constraints to find out the stationary points of free (non-constrained) function of $n+k$-variables: it introduces a new (unknown) scalar variable, the Lagrange multiplier, for each constraint present in the problem defining a new function (the Lagrangian) in terms of the original function, the constraints and the Lagrange multipliers. Up to this point, the Lagrange multipliers have been imposed a priori to modify the dynamics and select the form of the effective potential. Furthermore, by integrating the multipliers, cosmological solutions have been achieved. On the other hand, it is possible to show that the Lagrange multipliers are constraints capable of reducing the dynamics in higher order theories. Technically they are anholonomic constraints being time-dependent. They give rise to field equations which describe the dynamics of the further degrees of freedom coming from higher order theories. This fact is relevant to deal with such new degrees of freedom under the standard of effective scalar fields.

With these considerations in mind, let us take into account generic higher-order theories described by the action

$$
\mathcal{A}=\int d^{4} x \sqrt{-g} F\left(R, \square R, \square^{2} R, \ldots, \square^{k} R\right) .
$$

The field equations are

$$
\begin{aligned}
R^{\mu \nu}-\frac{1}{2} g^{\mu \nu} R & =\frac{1}{\mathcal{G}}\left\{\frac{1}{2} g^{\mu \nu}(F-\mathcal{G} R)+\left(g^{\mu \lambda} g^{\nu \sigma}-g^{\mu \nu} g^{\lambda \sigma}\right) \mathcal{G}_{; \lambda \sigma}+\right. \\
& +\frac{1}{2} \sum_{i=1}^{k} \sum_{j=1}^{i}\left(g^{\mu \nu} g^{\lambda \sigma}+g^{\mu \lambda} g^{\nu \sigma}\right)\left(\square^{j-i}\right)_{; \sigma}\left(\square^{i-j} \frac{\partial F}{\partial \square^{i} R}\right)_{; \lambda}+ \\
& \left.-g^{\mu \nu} g^{\lambda \sigma}\left[\left(\square^{j-1}\right)_{; \sigma} \square^{i-j} \frac{\partial F}{\partial \square^{i} R}\right]\right\},
\end{aligned}
$$

where

$$
\mathcal{G}=\sum_{j=0}^{k} \square^{j}\left(\frac{\partial F}{\partial \square^{j} R}\right)
$$

These are pure gravity $(2 k+4)$-order field equations. Matter can be taken into account by introducing, as above, the energy-momentum tensor $T_{\mu \nu}$.

In order to better discuss the role of Lagrange multipliers, let us consider, for the moment, actions containing up to $\square R$ terms. In this case, we have eight-order field equations which becomes of sixth-order if the theories is linear in $\square R$. If we take into account FRW point-like actions, we can reduce to the Lagrangian

$$
\mathcal{L}=\mathcal{L}(a, \dot{a}, R, \dot{R}, \square R,(\square R)),
$$

by which one can deduce the Euler-Lagrange equations corresponding to the Friedmann equations of cosmology. It is easy to show that such cosmological equations follow from Einstein gravity so deriving field equations from a field action and then reducing them to cosmological equations or reducing the field Lagrangian to a point-like Lagrangian and then deducing the Euler-Lagrange equations gives exactly the same results [12]. In Eq.(77), dot represents derivative with respect to cosmic time and, as standard for cosmological Lagrangian deduced from field theories, the covariance is lost. The variables $R$ and $\square R$ can be considered independent and, by the method of Lagrange multipliers, we can eliminate higher than one time derivatives. If we would not consider Lagrange multipliers, the Lagrangian (77) cannot be considered canonical[13]. The action related to Lagrangian (74), up to $\square R$ terms becomes

$$
\mathcal{A}=2 \pi^{2} \int d t\left\{a^{3} F-\lambda_{1}\left[R+6\left(\frac{\ddot{a}}{a}+\left(\frac{\dot{a}}{a}\right)^{2}+\frac{k}{a^{2}}\right)\right]-\lambda_{2}\left[\square R-\ddot{R}-3\left(\frac{\dot{a}}{a}\right) \dot{R}\right]\right\} .
$$

$\lambda_{1,2}$ are given by varying the action with respect to $R$ and $\square R$, that is

$$
\lambda_{1}=a^{3} \frac{\partial F}{\partial R}, \quad \lambda_{2}=a^{3} \frac{\partial F}{\partial(\square R)} .
$$


Only in this case the action results canonically defined in terms of $R$ and $\square R$ considered as independent variables. After an integration by parts, the point-like Lagrangian results

$$
\mathcal{L}=6 a \dot{a}^{2} \frac{\partial F}{\partial R}+6 a^{2} \dot{a} \frac{d}{d t}\left(\frac{\partial F}{\partial R}\right)-a^{3} \dot{R} \frac{d}{d t}\left(\frac{\partial F}{\partial(\square R)}\right)+a^{3}\left[F-\left(R+\frac{6 k}{a^{2}}\right) \frac{\partial F}{\partial R}-\square R \frac{\partial F}{\partial(\square R)}\right],
$$

where, clearly, the canonically conjugate variable of configuration space are the set $\mathcal{Q}=\{a, R, \square R\}$ and the relative velocities. A remark is necessary at this point. We can also take into account

$$
\lambda_{1}=a^{3}\left[\frac{\partial F}{\partial R}+\square \frac{\partial F}{\partial(\square R)}\right]
$$

as a Lagrange multiplier. The Lagrangian which comes out differs from (80) just for a term vanishing on the constraint, being

$$
\tilde{\mathcal{L}}=\mathcal{L}-a^{3}\left\{R+6\left[\frac{\ddot{a}}{a}+\left(\frac{\dot{a}}{a}\right)^{2}+\frac{k}{a^{2}}\right]\right\} \square \frac{\partial F}{\partial(\square R)} .
$$

From this point of view, considering the point-like Lagrangian $\mathcal{L}$ or $\tilde{\mathcal{L}}$ is completely equivalent.

It is important to stress that Lagrange multipliers are constraints that, after variation, give rise to further equations of motion (one for any multipliers). In fact, the expression that are multiplied by Lagrange multipliers in the action are constraints. The variation of the action with respect to the Lagrange multipliers gives equations of motion of the form "constraint equal to zero". Alternatively, one can solve the Lagrange multipliers and insert them into the action. From the resulting action one obtains, of course, the same constraint equations. To show this point, let us derive the EulerLagrangian equations from the Lagrangian (80) that is defined on the tangent bundle $\mathcal{T} \mathcal{Q} \equiv\{a, \dot{a}, R, \dot{R}, \square R,(\square R)\}$. They can be also deduced from the Einstein equations (75). The equation for the variables $\{a, \dot{a}\}$ gives

$$
\begin{aligned}
& {\left[R \frac{\partial F}{\partial R}+\square R \frac{\partial F}{\partial(\square R)}-F\right]+2\left[3 H^{2}+2 \dot{H}+\frac{k}{a^{2}}\right] \frac{\partial F}{\partial R}+} \\
+ & 2[\square R-H \dot{R}] \frac{\partial^{2} F}{\partial R^{2}}+\dot{R}(\square R) \frac{\partial^{2} F}{\partial(\square R)^{2}}+\left[2 \square^{2} R-2 H(\square R)+\dot{R}^{2}\right] \frac{\partial^{2} F}{\partial R \partial(\square R)}+ \\
+ & 2 \dot{R}^{2} \frac{\partial^{3} F}{\partial R^{3}}+2(\square R)^{2} \frac{\partial^{3} F}{\partial R \partial(\square R)^{2}}+4 \dot{R}(\square R) \frac{\partial^{3} F}{\partial R^{2} \partial(\square R)}=0 .
\end{aligned}
$$

The equation for $\{R,(\dot{R})\}$ gives

$$
\square \frac{\partial F}{\partial(\square R)}=0
$$

Finally, the equation for the pair $\{\square R, \square R\}$ coincides with the Lagrange multipliers

$$
\square R=\ddot{R}+3 H \dot{R}, \quad R=-6\left(\dot{H}+2 H^{2}+\frac{k}{a^{2}}\right) .
$$

The energy condition, that is the $(0,0)$-Einstein equation, gives

$$
H^{2}\left(\frac{\partial F}{\partial R}\right)+H \frac{d}{d t}\left(\frac{\partial F}{\partial R}\right)+\frac{1}{6}\left[\left(R+\frac{6 k}{a^{2}}\right) \frac{\partial F}{\partial R}+\square R \frac{\partial F}{\partial(\square R)}-F-\dot{R} \frac{d}{d t}\left(\frac{\partial F}{\partial(\square R)}\right)\right] .
$$

This derivation cleary show that dynamics can be made canonical by Lagrange multipliers. However, considering further higher-order $\square^{k} R$ terms the process can be made iterative since for each 2-orders (i.e. $\square$ ) one has another Lagrange multiplier. This method allows to select suitable changes of variables that can be identified once the variables $a, R$ and $\square R$ are disentangled [12]. As a consequence, dynamics can be reduced and exactly integrated [14, 15].

An important remark is in order at this point. If we take into account a conformal transformation as (31), it is easy to show that

$$
\tilde{g}_{\mu \nu} \equiv\left(\frac{d F}{d R}\right) g_{\mu \nu}, \quad \phi=\sqrt{\frac{3}{2}} \ln \left(\frac{d F}{d R}\right)
$$


for a $F(R)$-gravity and

$$
\tilde{g}_{\mu \nu} \equiv\left(\frac{\partial F}{\partial R}+\square \frac{\partial F}{\partial \square R}\right) g_{\mu \nu}, \quad \phi=\sqrt{\frac{3}{2}} \ln \left(\frac{\partial F}{\partial R}+\square \frac{\partial F}{\partial \square R}\right),
$$

for $F(R, \square R)$-gravity. It is easy to see that such transformations are related to the Lagrange multipliers (79) and (81). This means that operating a conformal transformation from the Jordan frame to the Einstein frame, that is disentangling the additional gravitational degrees of freedom related to higher order theories of gravity has the same dynamical meaning of reducing the dynamical system by imposing Lagrange multipliers.

\section{CONCLUSIONS}

In summary, we studied the role of Lagrange multiplier constraint for DE cosmology in scalar-tensor and quintessence theory and modified $F(R)$-gravity. It is demonstrated that the presence of constraint significally simplifies the reconstruction scenario. Moreover, the details of cosmological evolution are qualitatively changed. For instance, the phantom/non-phantom transition in scalar-tensor theory is more smooth. In modified gravity, the presence of Lagrange multiplier induces the necessity to introduce second $F(R)$ function which plays major role for cosmology. Again, the reconstruction program is qualitatively simplified. It is shown that viable reconstruction may be achieved. For instance, the examples of dark energy era and unified early-time inflation with late-time acceleration are worked out. We considered the constraint of specific form which permits to change the EoS parameter of the effective scalar fluid. However, many other choices for constraint may be useful. For instance, additional modification of Lagrange multiplier constraint may help to pass the local tests for the theory which cannot pass local tests in its original formulation.

A general comment is in order at this point. Gravitational theories are constrained theories. Such constraints can be anholonomic and then can result as further equations of motion for the related dynamical systems. We have first investigated the possibility that introducing by hand Lagrange multipliers, we can select suitable forms of the effective potential $V(\phi)$ and of $F(R)$-gravity. This approach leads to solvable DE dynamics for several physically interesting models. Besides, we have considered higher-order models. Lagrange multipliers allow, in this case, to make the theory canonical, that is they allow to disentangle the degrees of freedom of the problem and then reduce the dynamics. In some examples that we have worked out, it is possible to achieve exact solutions thanks to the multipliers that $i$ ) result as new equations of motion $i i$ ) allow suitable change of variables which, identifying cyclic variables, are related to constant of motion and allow to reduce and integrate the dynamics. The two methods (i.e. imposing the multipliers a priori or using them to reduce dynamics and make it canonical) are effectively equivalent and show the possibility to develop a new approach to alternative theories of gravity.

An important consideration is in order for conformal transformations. In the Einstein frame, gravitational degrees of freedom and scalar field are well separated. Imposing Lagrange multipliers means to search for suitable forms of the scalar field potential that allow to integrate dynamics. In this case, the Lagrange multipliers act as a "selection rule" on the dynamics and give fixed stationary points. On the other hand, in the Jordan frame, dynamics is not "canonical" since gravitational degrees of freedom and/or scalar fields are not disentangled. Lagrange multipliers, as said, make dynamics canonical. It is interesting to see that the form of conformal transformation can be related to the Lagrange multipliers (they have the same forms, see Eqs. (79), (81), (87), and (88)). In some sense we can state that imposing Lagrange multipliers in the Jordan frame and performing conformal transformations to the Einstein frame are the same operation. Also the reverse statement holds: given a Lagrangian in the Einstein frame endowed with Lagrange multipliers means, under conformal transformation to the Jordan frame, to take into account higher order or non-minimally coupled theories that are, in any case, canonical. This question will be investigated elsewhere.

\section{Acknowledgments}

This work has been suported by INFN-MEC collaboration project. The work by S.N. is supported in part by Global COE Program of Nagoya University provided by the Japan Society for the Promotion of Science (G07) and that by S.D.O. is supported in part by MEC (Spain) project FIS2006-02842 and LRSS project 3558.2010.2.

[1] E. A. Lim, I. Sawicki and A. Vikman, arXiv:1003.5751 [astro-ph.CO]. 
[2] C. Gao, Y. Gong, X. Wang and X. Chen, arXiv:1003.6056 [astro-ph.CO].

[3] S. Nojiri and S.D. Odintsov, arXiv:1004.3613

[4] S. Nojiri and S. D. Odintsov, Gen. Rel. Grav. 38, 1285 (2006) arXiv:hep-th/0506212.

[5] S. Nojiri, S. D. Odintsov and S. Tsujikawa, Phys. Rev. D 71, 063004 (2005) arXiv:hep-th/0501025.

[6] R. R. Caldwell, Phys. Lett. B 545, 23 (2002) arXiv:astro-ph/9908168;

R. R. Caldwell, M. Kamionkowski and N. N. Weinberg, Phys. Rev. Lett. 91, 071301 (2003) arXiv:astro-ph/0302506;

B. McInnes, JHEP 0208 (2002) 029 arXiv:hep-th/0112066;

S. Nojiri and S. D. Odintsov, Phys. Lett. B 562, 147 (2003) arXiv:hep-th/0303117;

S. Nojiri and S. D. Odintsov, Phys. Lett. B 571, 1 (2003) arXiv:hep-th/0306212;

V. Faraoni, Int. J. Mod. Phys. D 11, 471 (2002) arXiv:astro-ph/0110067;

P. F. Gonzalez-Diaz, Phys. Lett. B 586, 1 (2004) arXiv:astro-ph/0312579;

M. Sami and A. Toporensky, Mod. Phys. Lett. A 19, 1509 (2004) arXiv:gr-qc/0312009;

H. Stefancic, Phys. Lett. B 586, 5 (2004) arXiv:astro-ph/0310904);

L. P. Chimento and R. Lazkoz, Phys. Rev. Lett. 91, 211301 (2003) arXiv:gr-qc/0307111.

[7] S. Capozziello, S. Nojiri and S. D. Odintsov, Phys. Lett. B 632, 597 (2006) arXiv:hep-th/0507182.

[8] S. Capozziello, S. Nojiri, S. D. Odintsov and A. Troisi, Phys. Lett. B 639, 135 (2006) arXiv:astro-ph/0604431;

S. Nojiri and S. D. Odintsov, Phys. Rev. D 74, 086005 (2006) arXiv:hep-th/0608008.

[9] T. Chiba, T. Okabe and M. Yamaguchi, Phys. Rev. D 62, 023511 (2000) arXiv:astro-ph/9912463;

C. Armendariz-Picon, T. Damour and V. F. Mukhanov, Phys. Lett. B 458, 209 (1999) arXiv:hep-th/9904075.

[10] J. Matsumoto and S. Nojiri, Phys. Lett. B 687, 236 (2010) arXiv:1001.0220 [hep-th]].

[11] S. Nojiri and S. D. Odintsov, Phys. Rev. D 68, 123512 (2003) arXiv:hep-th/0307288;

S. Nojiri and S. D. Odintsov, eConf C0602061, 06 (2006) [Int. J. Geom. Meth. Mod. Phys. 4, 115 (2007)] arXiv:hep-th/0601213;

Applications. S. Capozziello and M. Francaviglia, Gen. Rel. Grav. 40, 357 (2008) arXiv:0706.1146 [astro-ph]].

[12] S. Capozziello, R. De Ritis, C. Rubano and P. Scudellaro, Riv. Nuovo Cim. 19N4, 1 (1996).

[13] M. Chaichian and A. Demichev, Path Integrals in Physics, Vol. I and II, Institute of Physics Publishing, 2001, Bristol and Philadelphia;

M. Henneaux and C. Teitelboim, Quantization of Gauge Systems, Princeton University Press, 1994, Princeton, New Jersey.

[14] S. Capozziello and A. De Felice, JCAP 0808, 016 (2008) arXiv:0804.2163 [gr-qc]].

[15] S. Capozziello, P. Martin-Moruno and C. Rubano, Phys. Lett. B 664, 12 (2008) arXiv:0804.4340 [astro-ph]]. 